\title{
La visualización de autores en un repositorio institucional a través del enfoque Model Driven con WebRatio
}

\author{
The visualization of authors in an Institutional Repository through the Model Driven approach with WebRatio
}

\author{
Jose Texier (1), Marisa R. de Giusti, Gonzalo L. ViLLARreal y Ariel J. LiRA (2)
}

(1) Universidad Nacional Experimental del Táchira (UNET), Venezuela; jtexier@unet.edu.ve; Universidad Nacional de Chilecito (UNdeC), Argentina, jtexier@undec.edu.ar. (2) Marisa R. De Giusti, Gonzalo L. Villarreal y Ariel J. Lira, Servicio de Difusión de la Creación Intelectual, Universidad Nacional de La Plata (SEDICI), Argentina, \{marisa.degiusti, gonzalo, ariel\} @sedici.unlp.edu.ar

\section{Resumen}

Se plantea una metodología de desarrollo de software para visualizar y modificar los autores de los recursos de tipo "Tesis" en el Repositorio Institucional de la Universidad Nacional de La Plata, Argentina. Se aplicó la metodología Model-Driven. Además de resolver el problema, la metodología ayudó a las partes involucradas (desarrolladores, dueños del negocio y expertos del dominio) a interactuar en un mismo idioma sin importar el software de repositorios usado, por lo que tiene interés general.

Palabras clave: Repositorios institucionales universitarios. Model-driven. Webratio. Autores. Control de autoridades. Universidad Nacional de La Plata.

\section{Introduction}

Los trabajos científicos, académicos y administrativos de instituciones se recopilan, catalogan, acceden, gestionan, difunden y preservan en el tiempo a través de sistemas informáticos conocidos como Repositorios Institucionales (RI) los cuales están relacionados con los ideales y objetivos del Acceso Abierto (Björk y Solomon, 2012; Suber, 2012). Los RI funcionan a partir de la enumeración de un conjunto de datos específicos de diversos trabajos llamados metadatos (Texier, 2013). Además, se desarrollan mediante plataformas de software que permiten gestionar los diferentes servicios que prestan. Algunas instituciones utilizan plataformas preexistentes, las más usadas son DSpace, EPrints y Digital Commons (OpenDOAR, 2015; ROAR, 2015), mientras que otras realizan desarrollos propios, sobre los cuales se gestiona un gran porcentaje de los recursos depositados, a saber: arXiv, CiteSeerX, Social Science Research Network, entre otras (CSIC, 2015).

Todas las plataformas de software son diseñadas e implementadas bajo alguna metodología de desarrollo de software que garantice el mantenimiento, escalabilidad, performancia, etc. ante el manejo de grandes datos.

\begin{abstract}
A methodology for software development is offered that allows repository administrators to view and modify the authors of the resource type "Theses". It was developed for the Institutional Repository of the National University of La Plata, Argentina, applying the Model-Driven methodology. In addition to solving the problem, this methodology helped the involved stakeholders (developers, business owners and domain experts) to interact with the same language regardless of the used software, so this methodology is easily generalizable to other similar problems.
\end{abstract}

Keywords: Institutional repositories. Universities. Model-driven. Webratio. Authors. Authority control. Universidad Nacional de La Plata.

En ese contexto coexisten distintos factores que determinan el diseño e implementación de los componentes de software en un repositorio para buscar soluciones a sus problemas (Texier y De Giusti, 2014): la diversidad de soluciones tecnológicas, el tratamiento de diferentes tipologías de recursos, los esquemas de metadatos, las recomendaciones de almacenamientos de recursos, la preservación de recursos y las recomendaciones de modelos conceptuales o de datos como: OAIS, FRBR, modelo 5S, modelo DELOS, Europeana Data Model, entre otros.

Estos factores se pueden contemplar dentro de metodologías de desarrollo dirigidas por modelos para generar una aplicación web que solucione algún problema mediante la plataforma de software WebRatio (WebRatio, 2015). Por tanto, el objetivo del estudio fue visualizar y modificar los autores de los recursos de tipo "Tesis" en SEDICI, que es el Repositorio Institucional de la Universidad Nacional de La Plata (SEDICI, 2015), para dar una solución que permitiera abordar el problema en los RI sin tener que comprender la plataforma de software y permitir a los distintos actores en este sistema de software (desarrolladores, dueños del negocio y expertos del dominio) proponer soluciones e 
ideas independientes de la plataforma subyacente, es decir, manejar el mismo idioma desde el punto de vista del diseño y el desarrollo de soluciones informáticas.

Este artículo está organizado de la siguiente manera: una exposición del problema; luego, la metodología de desarrollo de software dirigida por modelos para llevar a cabo la implementación de la propuesta; después se presenta la propuesta, el diseño, el análisis y la implementación realizada; y, finalmente, se presentan las conclusiones.

\section{El problema}

Los recursos, dentro del contexto de los RI, son objetos físicos o digitales que se describen a partir de la enumeración de un conjunto de datos específicos (metadatos) que lo distinguen entre otros objetos. Estos metadatos son representados a través de distintos modelos, esquemas, formatos o estándares, que si bien comparten una sintaxis y estructura de la información, por lo general en XML (W3C, 2015), difieren a los propósitos de la información que describen (Méndez, 2003). La entidad abstracta autor en SEDICI, es el metadato de los recursos que permite identificar el creador intelectual del recurso, ya sea una persona o una organización. El autor se considera entidad abstracta, en SEDICI, por poseer información descriptiva propia, utilizada en los procesos de catalogación de recursos como elemento de un vocabulario controlado (Texier et al., 2013). Ante esto, se entiende la importancia de localizar el autor de un recurso para identificarlo, a través de búsquedas o navegación en la plataforma.

Los autores son catalogados de diferentes maneras en los software de repositorios, ya que existen múltiples formas de normalizar dicha entidad para el funcionamiento e interoperabilidad de los RI en el mundo. Por ello, es necesario tener un control completo de tal entidad (autores), de esta manera cualquier plataforma de software podrá gestionar la normalización de la forma que desee. Para lograrlo es recomendable que un autor tenga un registro lo más completo posible en el que incluya los datos y nombres completos lo que será mejor para la gestión del RI. A su vez, es necesario contar con una interfaz que permita la gestión de tales datos. En otras palabras, la entidad autor es estandarizada (en SEDICI) para lograr su identificación, recuperación y uso, de esa manera se evita la redundancia y se garantiza la integridad de la información. Se consideran tres puntos de vista de los problemas presentes en el proceso de catalogación (De Giusti et al., 2011) que pueden afectar dicha entidad abstracta:

- Forma de representación: depende del esquema de metadatos seleccionado, similar a los problemas presentes en los vocabularios controlados.

- Forma de referenciar: una vez seleccionada una entidad abstracta es necesario guardar la referencia, lo cual trae problemas de compatibilidad entre la representación elegida para la entidad abstracta y los metadatos del recurso a los cuales se asocia esa entidad.

- Forma de presentación: es como el usuario observará y utilizará los metadatos en el portal del repositorio, por ejemplo: formularios de carga, página de presentación de metadatos, exportación de recursos, etc. Puede ser simple, intuitiva, e internacionalizable, pero considerando los problemas generados en el formato de catalogación usado se tienen dos alternativas: en el momento de catalogación debe realizarse una transformación única que elimine el problema de duplicidad y de consistencia; y en el momento de presentación se requiere de una transformación cada vez que se muestre el recurso. Esto implica una mayor carga de procesamiento (evitar duplicidad y asegurar consistencia).

Estos problemas son solventados en este trabajo gracias a la metodología Model-Driven, incorporada en WebRatio. WebRatio es una herramienta soportada por una metodología de desarrollo dirigida por modelos, que permitió visualizar y modificar los autores de SEDICI (2015) y crearle sus respectivas funciones como el ABM (alta, baja y modificación). SEDICI usa DSpace como plataforma de software (Dspace, 2015) que ofrece dos intefaces (XMLUI y JSPUI) y deja abierta la gestión de la entidad autores. SEDICI decidió gestionar la entidad autores de la misma forma como la realizaba en la plataforma de software de la que migró en el 2012 (Celsius DL) y que está basada en una base de datos MySQL. Entonces, WebRatio, brinda la posibilidad de relacionar el DSpace de SEDICI con la base de datos de autores en MySQL como una alternativa más de todas las opciones existentes.

\section{Metodología de desarrollo de software}

Los actores que intervienen en el diseño e implementación de un sistema de software (desarrolladores, dueños del negocio y expertos del dominio) tomaron conciencia de la necesidad de formalizar la construcción de componentes de software y guiar tales desarrollos a través de 
modelos. Estos son definidos como un conjunto de elementos que sirven para demostrar la consistencia de una teoría, es decir, representan con detalle un sistema dado (Whittle et al., 2011). Este enfoque es conocido como ModelDriven, del cual nace el Model Driven Software Engineering (MDSE), que define una metodología de desarrollo de software para aplicar las ventajas del modelado en actividades de ingeniería de software (Brambilla et al., 2012).

MDSE se puede aplicar en diferentes niveles de abstracción para proporcionar una visión integral en el desarrollo del sistema de software. En esta metodología los modelos se transforman en otros modelos de manera sucesiva hasta obtener una representación final (artefactos de software o código fuente), gracias a la definición de un lenguaje que proporciona la posibilidad de describirlos adecuadamente (Navarro et al., 2011).

WebRatio, herramienta de software usada en este trabajo, tiene su base en una metodología de modelado Web orientada a datos que lleva por nombre WebML (Acerbis et al., 2008; Ceri et al., 2000) y corresponde al dominio ModelDriven Engineering (MDE). WebRatio es un software comercial (con licencias para desarrollo comercial, personal y académico) usado para el diseño y la implementación de aplicaciones web y está soportado por el ambiente de desarrollo de software integrado Eclipse (WebRatio, 2015). Los requisitos se expresan a través de un modelo de alto nivel y el código de la aplicación se genera automáticamente (mediante el uso de reglas). El código resultante es una aplicación en el lenguaje de programación Java y basada en estándares Web. Consta de tres pasos básicos: construcción de los modelos (en particular, el modelo de la aplicación se realiza utilizando WebML), personalización de las reglas y generación de la aplicación (Brambilla y Fraternali, 2013).

\section{Desarrollo de la propuesta}

El ideal para los actores involucrados es que exista un lenguaje neutral y de alto nivel que permita que las partes se pongan de acuerdo y les sirva de apoyo para describir, discutir y negociar las funciones (recopilar, catalogar, almacenar, gestionar, acceder, difundir y preservar) que el Repositorio debe ofrecer (Texier et al., 2012). El enfoque Model-Driven brinda el marco necesario a los interesados para compartir sus puntos de vista y manipular directamente las representaciones de las entidades de este dominio. Además, este paradigma ofrece algunas ventajas como: incremento en la productividad (errores, costos, código), adaptación a cambios tecnológicos, reuso de software, mejora en la comunicación con usuarios y desarrolladores, asignación de roles, entre otros (Pons et al., 2010). Por ello, a partir de un repositorio funcionalmente activo (SEDICI) y siguiendo la metodología MDSE a través de WebRatio se desarrollaron las siguientes funcionalidades, entre otras:

- visualización de los elementos de una representación de recursos del repositorio,

- interoperabilidad entre las plataformas de software,

- evaluación de la correlación de entidades con cualquier esquema de metadatos,

- análisis de los metadatos que garantizan la preservación de los recursos,

- almacenamiento de las entidades en cualquier tipo de base de datos,

- establecimiento de métricas relacionadas a entidades deseada (p.e.: descargas, visitas, búsquedas),

- sistemas científicos más inteligentes,

- coherencia con los conceptos de la norma ISO 14721, que ofrece una recomendación a la administración de documentos para poder especificar la representación de un recurso dentro de un RI,

- tareas de curación de forma rápida y sencilla de un RI.

Estas funcionalidades pueden implementarse a partir de un enfoque MDSE con independencia tecnológica, es decir, puede adaptarse a diferentes plataformas de software. Por razones de espacio, este trabajo se centra únicamente en la visualización y modificación de la entidad autor en SEDICI para los recursos de tipo tesis (grado, especialización, maestría y doctorado) pertenecientes a la Universidad Nacional de La Plata (UNLP). Se crearon las siguientes funciones para la entidad autores diseñadas con componentes de software en WebRatio:

- Funciones básicas de crear, leer, actualizar y borrar (ABM).

- Exportar e importar registros en archivos $\mathrm{XML}$, a partir de esquemas de definición en XML (XSD).

- Modificación de la entidad autores, a través de consultas SQL (por lotes).

- Búsquedas y consultas a la base de datos, gracias a sentencias SQL. 
- Control de acceso de los usuarios para modificar, gracias al soporte de seguridad que ofrece WebRatio.

\subsection{Análisis}

A partir de WebRatio se diseñaron los diferentes modelos involucrados en el proceso, los cuales se compartieron con los diferentes actores de SEDICl. La institución, para el 2014, contaba con aproximadamente 41.000 recursos digitales catalogados uno por uno por expertos en el área desde el 2003. El desarrollo actual de SEDICI se encuentra en Dspace, pero la base de datos de los autores está en MySQL debido a que la versión anterior del repositorio era un desarrollo propio (Celsius DL) que se migró a DSpace en el 2012, aprovechando así el soporte que brinda DSpace para un control de autoridades abierto. Por tanto, se tienen dos bases de datos en WebRatio, una en PostgreSQL (DSpace) y una en MySQL (Celsius DL). Es importante destacar que la intención fue diseñar e implementar funcionalidades en WebRatio a partir de la base de datos de SEDICl, en ningún momento se desarrolló otra base de datos y no se tuvo redundancia de datos, ya que el eje central era visualizar y modificar los autores del repositorio a través de un proceso que sirviera de base a otros repositorios o sistemas similares.

\subsection{Diseño e implementación}

WebRatio exige dos modelos básicos para generar la aplicación Web que se desea: un modelo de datos y un modelo de hipertexto, pero antes de ellos, es conveniente realizar un diseño de cómo se quiere la interfaz de la aplicación. Para este trabajo se diseñaron 3 modelos de interfaz correspondientes a la visualización de los autores, tesis y ABM de los autores. Se presentará el modelo de la interfaz de autores en la Figura 1. En esta figura, se observa el prototipo de interfaz y se definen los distintos frames de trabajo, los cuales se irán incorporando en las diferentes capas de diseño y desarrollo en WebRatio.

En cuanto al modelo de datos, se importaron de la base de datos de SEDICI (DSpace-SEDICI y Celsius DL) que contiene los registros de los 41.000 recursos y 19.500 autores y usuarios, respectivamente. A continuación se muestra una parte del modelo de hipertexto desarrollado (Figura 2) conocido en WebRatio como SiteView, en caso de querer profundizar en los modelos y el proyecto WebRatio, pueden visitar el proyecto GitHub (Texier, 2014) en el que se encuentra todo el código fuente del trabajo realizado.

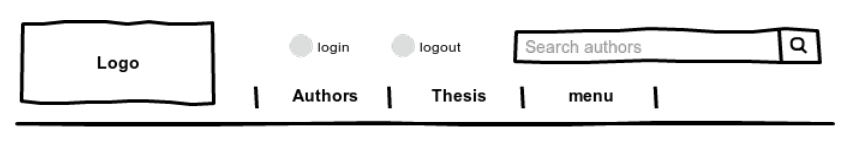

Authors list
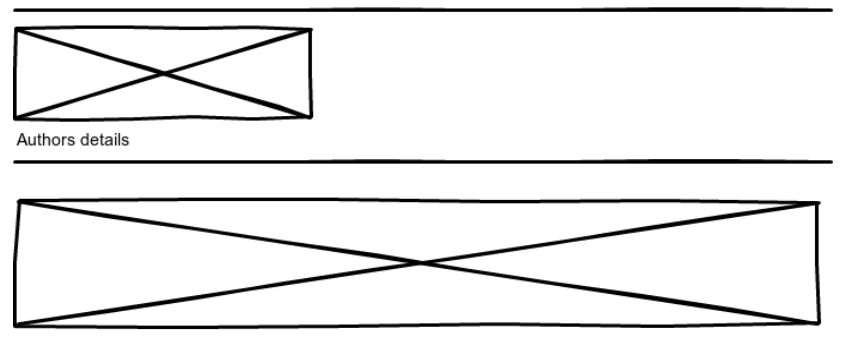

Thesis for authors

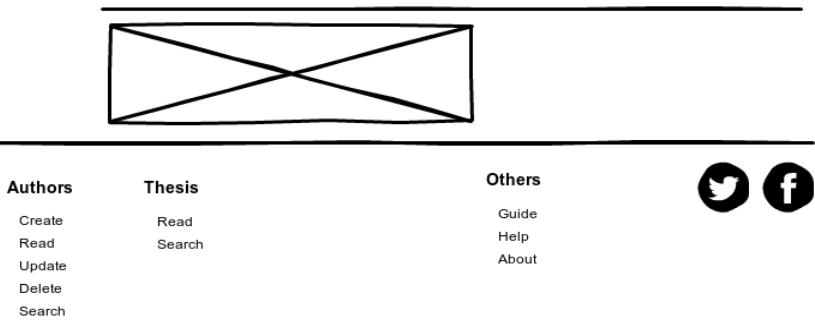

Figura 1. Modelo de la interfaz de autores

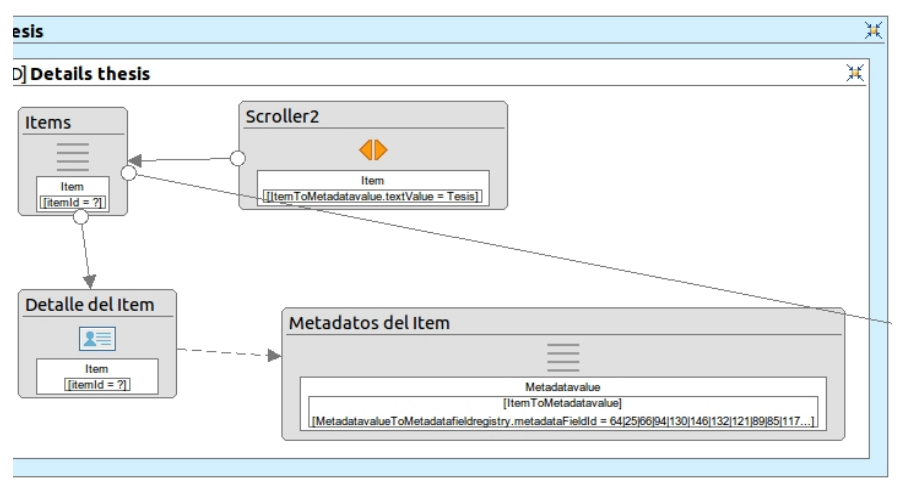

Figura 2. Modelo hipertexto

Luego de la sincronización de las bases de datos a través del modelo de datos y de hipertexto (SiteView) en WebRatio, el desarrollador del sistema da clic al botón de generación de código y levanta el servidor de aplicaciones Web. Luego, cualquier usuario dentro del contexto de la red donde se encuentra montada la aplicación, despliega el navegador Web donde se observa la aplicación elaborada (Figura 3).

\section{Ventajas de Model-Driven en los repositorios}

El trabajo realizado sirve de base para implementar alguna funcionalidad de los repositorios sin modificar los componentes de software en el sistema de repositorios. Asimismo, permitió entender el proceso básico del desarrollo de software aplicado y lo beneficioso que es para los tres actores presentes del dominio de los 
repositorios (desarrolladores, dueños del negocio y expertos del dominio) gracias al paradigma Model-Driven (Texier et al., 2014). A continuación se mencionan los beneficios para cada uno de los actores que intervienen en el proceso.
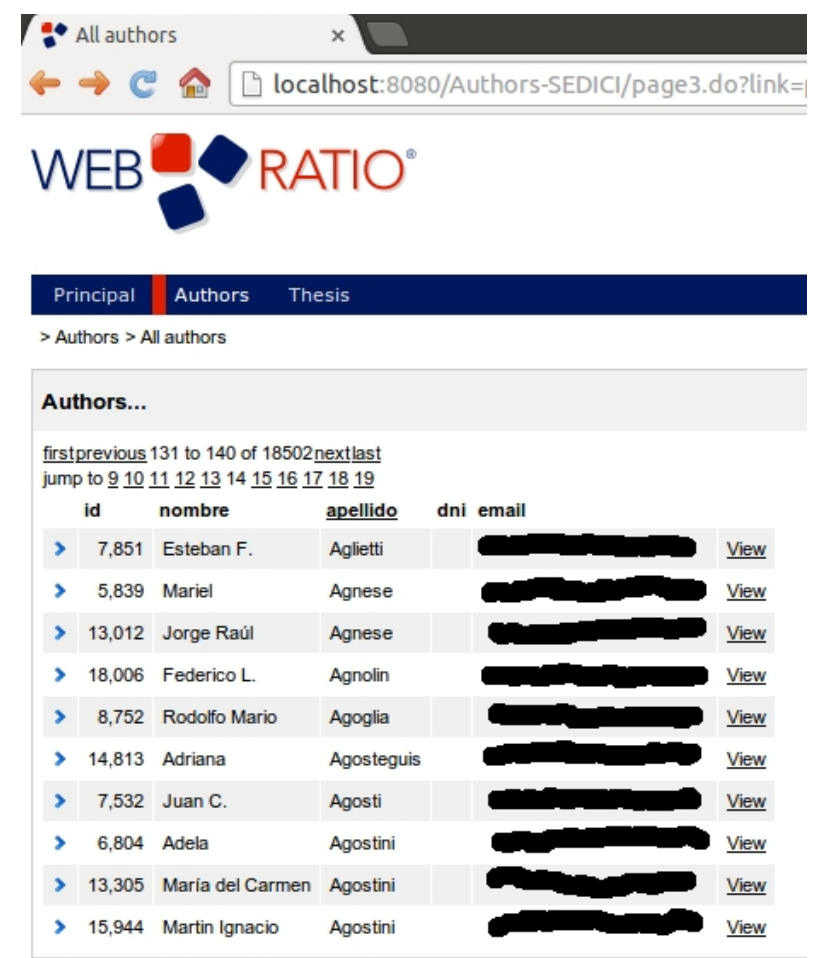

Figura 3. Site Web generado automáticamente

\subsection{Los desarrolladores}

Los desarrolladores, también conocidos como diseñadores, son responsables de plasmar en código ejecutable los requerimientos del sistema a desarrollar:

- Obtener menor número de líneas de código escritas, gracias a los niveles de abstracción del enfoque Model-Driven y se minimizan: tasa de errores, tareas de mantenimiento y de mejoramiento.

- Lograr un alto nivel de abstracción para escribir aplicaciones y componentes de software a través de la arquitectura del Model-Driven.

- Generar una especificación de requisitos de usuario a varios niveles mediante un sistema flexible a los cambios.

- Evitar la adopción de una única tecnología de hardware particular gracias a los niveles de abstracción.

- Garantizar la interoperabilidad entre los objetos en los sistemas de repositorios en un entorno multiplataforma.

\subsection{Los dueños del negocio}

Tienen la responsabilidad de coordinar y/o financiar el proyecto de desarrollo e implementación del sistema dentro de la organización o institución, es decir, los jefes de las unidades académicas donde se encuentra el repositorio, los beneficios son:

- Desarrollar componentes de software para los sistemas o funcionalidades específicas de los repositorios.

- Preservar digitalmente los recursos y/o los objetos generando estrategias que toman los niveles altos de abstracción de la metodología aplicada en el trabajo. La importancia de la preservación radica en garantizar la perpetuidad en el tiempo del recurso almacenado.

- Generar código para otras plataformas a futuro.

- Reducción de costes en el desarrollo de aplicaciones debido a la disminución del recurso humano requerido, de las horas hombre y del tiempo invertido en las diferentes actividades relacionadas.

- Documentar todo el proceso de desarrollo de software.

\subsection{Expertos del dominio}

Representan a los especialistas del dominio de los RI, por ejemplo, bibliotecarios, jefes de la dependencia responsable del repositorio, etc., los beneficios son:

- Permitir la revisión de modelos por parte de los distintos expertos del dominio. A diferencia de los desarrolladores quienes se concentran en los detalles técnicos.

- Generar lenguajes específicos del dominio en las fases de la implementación de los RI bajo Model-Driven, tales como: modelo de datos, modelo de la arquitectura, modelo de las entidades abstractas, interfaz de usuario, entre otros.

- Garantizar la interoperabilidad entre los distintas plataformas, ya que ellas pueden comunicarse y establecer lineamientos similares a partir de la metodología Model-Driven.

\section{Conclusiones}

El estudio realizado cumplió con el propósito de poder visualizar y modificar la entidad autores en SEDICl a través del enfoque Model Driven, es decir, resolvió un problema concreto mediante una determinada tecnología. Este enfoque 
ayuda a que las partes involucradas en un sistema de repositorios (desarrolladores, dueños del negocio y expertos del dominio) compartieran un mismo idioma sin importar la plataforma de software de repositorios. Por consiguiente, la metodología desarrollada puede ser aplicable sobre cualquier repositorio para implementar una funcionalidad deseada por los dueños o expertos del negocio.

De igual manera, se observó la utilidad que puede tener WebRatio en los repositorios institucionales, ya que se implementarían diversas funcionalidades sin importar la arquitectura de software en la que se encuentra el repositorio, de allí que la propuesta base se enfocara en la plataforma de software DSpace en SEDICI como campo de pruebas.

Los trabajos futuros que pueden llevarse a cabo luego del trabajo realizado son: aplicar la metodología desarrollada en otras plataformas de RI reconocidas para establecer comparaciones, realizar un estudio de las diferentes alternativas de herramientas de desarrollo de aplicaciones Web bajo el enfoque MDE e implementar otras funcionalidades que permitan tener un criterio más certero del alcance real de WebRatio.

\section{Referencias}

Acerbis, R.; Bongio, A.; Brambilla, M.; Butti, S.; Ceri, S.; Fraternali, P. (2008). Web Applications Design and Development with WebML and WebRatio 5.0. En: R.F. PAIGE y B. MEYER (eds.), Objects, Components, Models and Patterns. // Springer Berlin Heidelberg, Lecture Notes in Business Information Processing, 11, 392-411. ISBN 978-3-540-69823-4, 978-3-540-69824-1. http://link .springer.com/chapter/10.1007/978-3-540-69824-1_22.

Björk, B.-C.; Solomon, D. (2012). Open access versus subscription journals: a comparison of scientific impact. // PMID: 22805105, BMC Medicine. 10:1, 73. ISSN 17417015. DOI 10.1186/1741-7015-10-73.

Brambilla, M.; Cabot, J.; Wimmer, M. (2012). Model-Driven Software Engineering in Practice. // Morgan \& Claypool. ISBN 9781608458820.

Brambilla, M.; Fraternali, P. (2013). Large-scale ModelDriven Engineering of web user interaction: The WebML and WebRatio experience. // Science of Computer Programming. ISSN 0167-6423. DOI: 10.1016/j.scico. 2013.03.010. http://www.sciencedirect.com/science/articl e/pii/S0167642313000701.

Ceri, S.; Fraternali, P.; Bongio, A. (2000). Web Modeling Language (WebML): a modeling language for designing Web sites. // Computer Networks. 33:1-6, 137-157. ISSN 1389-1286. DOI 10.1016/S1389-1286(00)00040-2.

CSIC (2015). Ranking Web of Repositories. http://reposi tories.webometrics.info/. (12-05-2015).

De Giusti, M.; Oviedo, N.; Lira, A.; Sobrado, A.; Martinez, J.; Pinto, A. (2011). SEDICI - Desafíos y experiencias en la vida de un repositorio digital. RENATA. 1:2, 16-33. ISSN 2027-7415.
DSPACE (2015). DSpace. [en línea]. http://www.dspace. org/. (12-05-2015).

Méndez, E. (2003). Tratamiento de los objetos de información en los archivos: retos y estándares para la descripción basada en metadatos. http://eprints.rclis.org/handle/ 10760/12691\#.UAAZFuEzfgM.

Navarro, A.; Cristóbal, J.; Fernández-Chamizo, C.; Fernández-Valmayor, A. (2011). Architecture of a multiplatform virtual campus. // Software: Practice and Experience. ISSN 1097-024X. DOI: 10.1002/spe.1130. http://online library.wiley.com/doi/10.1002/spe.v42.10/issuetoc.

OPENDOAR (2015). OpenDOAR - Home Page - Directory of Open Access Repositories. http://www.opendoar.org/. (21-05-2015).

Pons, C.; Giandini, R.; Pérez, G. (2010). Desarrollo de Software Dirigido por Modelos. La Plata: Mc Graw Hill.

ROAR (2015). Registry of Open Access Repositories (ROAR). http://roar.eprints.org/. (21-02-2015).

SEDICI (2015). Repositorio de la Universidad Nacional de La Plata. http://sedici.unlp.edu.ar/.(14-06-2015).

Suber, P. (2012). Ensuring open access for publicly funded research. // PMID: 2287595, BMJ : British Medical Journal. 345. ISSN 0959-8138. DOI 10.1136/bmj.e5184. http://www.ncbi.nlm.nih.gov/pmc/articles/PMC3414432/.

Texier, J. (2013). Los repositorios institucionales y las bibliotecas digitales: una somera revisión bibliográfica y su relación en la educación superior. // 11th Latin American and Caribbean Conference for Engineering and Technology - 2013. Cancun, Mexico: LACCEI, 9. ISBN 10 9780-9822896-6-2. http://eprints.rclis.org/19925/.

Texier, J. (2014). WebRatio GitHub. [en línea]. http://github.com/dantexier/WebRatio. (21-02-2015).

Texier, J.; De Giusti, M. (2014). Elements of Resource Representation in Institutional Repositories: a Bibliographic Review. // Journal of Information and Organizational Sciences. 38:1. ISSN 1846-9418. https://jios.foi.hr/ index.php/jios/article/view/816.

Texier, J.; De Giusti, M. y Gordillo, S. (2014). Model-driven software development in the institutional repositories. // DYNA. 81:184, 7. ISSN 2346-2183.

Texier, J.; De Giusti, M.R.; Oviedo, N.; Lira, A.J.; Villarreal, G.L. (2013). La representación de recursos en los repositorios institucionales. El caso de estudio: SEDICI. // III Conferencia de Bibliotecas y Repositorios Digitales de América Latina (BIREDIAL) y VIII Simposio Internacional de Bibliotecas Digitales (SIBD) (Costa Rica, 2013). http://hdl.handle.net/10915/30111.

Texier, J.; De Giusti, M.R.; Oviedo, N.; Villarreal, G.L. y Lira, A.J. (2012). Los beneficios del desarrollo dirigido por modelos en los repositorios institucionales. // BIREDIAL - Conferencia Internacional Acceso Abierto, Comunicación Científica y Preservación Digital. http://hdl.handle. net/10915/26044.

W3C (2015). Extensible Markup Language (XML). [en línea]. http://www.w3.org/XML/. (20-01-2015).

WebRatio (2015). WebRatio. http://www.webratio.com/ portal/content/en/home. (20-01-2015).

Whittle, J.; Clark, T. y Kühne, T. (eds.)(2011). Model Driven Engineering Languages and Systems. // MODELS 2011. Wellington, New Zealand. Lecture Notes in Computer Science. http://www.springerlink.com/content/978-3-64224484-.

Enviado: 2014-11-26. Segunda versión: 2015-08-01. Aceptado: 2015-08-04 